\title{
A Simple Mechanism for Unconventional Superconductivity in a Repulsive Fermion Model
}

\author{
Kevin Slagle ${ }^{1,2^{*}}$, Yong Baek Kim ${ }^{1,3}$ \\ 1 Department of Physics, University of Toronto, Toronto, Ontario M5S 1A7, Canada \\ 2 Department of Physics and Institute for Quantum Information and Matter, \\ California Institute of Technology, Pasadena, California 91125, USA \\ 3 Canadian Institute for Advanced Research, Toronto, Ontario, M5G 1M1, Canada \\ * kslagle@caltech.edu
}

September 19, 2018

\begin{abstract}
We introduce a simple tight-binding lattice model of fermions with repulsive interactions that exhibits unconventional superconductivity. The Cooper pairing arises from a simple 4-site fermion model with repulsive interactions. In the strong coupling limit, the model reduces to a superconducting hard-core BoseHubbard model. Above the superconducting critical temperature, an analog of pseudo-gap physics results where the fermions remain Cooper paired with a large single-particle energy gap.
\end{abstract}

\section{Introduction}

Understanding unconventional superconductivity [1-8] arising from electron-electron interactions is a long-standing problem that has been thoroughly discussed in the context of cuprate [9-15] and iron-based [16-22] superconductors. While there exists a vast literature on this subject, the complexity of these materials is often an obstacle for theoretical modelling. As such, a simple toy model of unconventional (i.e. not phonon-mediated) superconductivity could be quite useful for strengthening our understanding of electron-interaction-driven superconductivity.

In general, the dominant contributions to an electron Hamiltonian can be modeled on a lattice by a generalized Hubbard model:

$$
H=-\sum_{I J} t_{I J} c_{I}^{\dagger} c_{J}+\sum_{I J} U_{I J} n_{I} n_{J}
$$

where $I$ and $J$ include position, orbital, and spin degrees of freedom. The electron hopping $t_{I J}$ describes the kinetic energy contribution, while the Hubbard interaction $U_{I J} \geq 0$ describes the repulsive Coulomb force. In order to superconduct, pairs of electrons must form a bosonic bound state, the so-called Cooper pair, which must then condense ${ }^{1}$. It may seem unnatural

\footnotetext{
${ }^{1}$ That is, the Hamiltonian must have ground states with $\langle b(x)\rangle \neq 0$, where $b(x) \sim \sum_{I J} \psi_{I J}(x) c_{I} c_{J}$ is a Cooper pair annihilation operator and $\psi_{I J}(x)$ is the superconducting order parameter. This occurs when the charge conservation symmetry $\left(c_{I} \rightarrow e^{i \theta} c_{I}\right)$ is spontaneously broken, as per Ginzburg-Landau theory. (As is commonly done, we are approximating the electrodynamic gauge field as a classical background field, rather than a dynamical field [23] which would be integrated over in the partition function.)
} 
for electrons to form a bound state due to repulsive interactions. Nevertheless, various mechanisms for how this could occur have been proposed in the literature, such as Fermi surface instabilities [24-32] inhomogeneity-induced superconductivity [33-37], and excitonic attraction [38-40].

In this work, we present a simple (and perhaps minimal) toy model of fermions with a repulsive interaction, which gives rise to a superconducting state with short coherencelength Cooper pairs in the strong coupling limit. The superconductivity mechanism is a manifestation of excitonic pairing based on the local physics of a 4-site spinless fermion model with a repulsive interaction.

We emphasize that our motivation is not to describe any current material. Rather, the motivation is to study a minimal model of unconventional superconductivity to gain physical intuition, which may be useful for designing or discovering new classes of superconducting materials.

\section{Superconductivity Mechanism}

A simple mechanism for the emergence of an effective attraction due to repulsive interactions can be understood from the following 4 -site spinless fermion model:

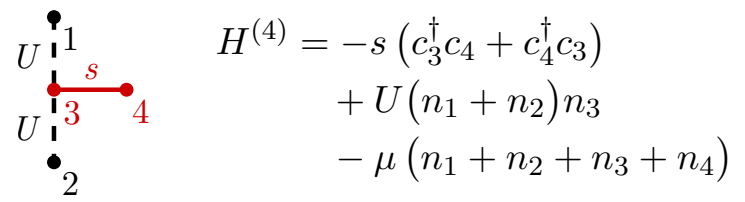

$c_{\alpha}$ are four spinless fermion annihilation operators with site/orbital index $\alpha=1,2,3,4$; $n_{\alpha}=c_{\alpha}^{\dagger} c_{\alpha}$ is the fermion number operator. $s$ is a fermion hopping strength; $U$ is a nearestneighbor Hubbard repulsion; and $\mu$ is the chemical potential.

When $\mu=s / 2$ and $s \ll U$, the two lowest energy levels of $H^{(4)}$ have the following eigenstates [up to corrections of order $O(s / U)$ ] and energies [up to $O\left(s^{2} / U\right)$ ]:

\begin{tabular}{ll|c} 
& $|\psi\rangle$ & $E$ \\
\hline$|\tilde{0}\rangle=$ & $\frac{1}{\sqrt{2}}\left(c_{3}^{\dagger}+c_{4}^{\dagger}\right)|0\rangle$ & $-\frac{3}{2} s$ \\
$|\tilde{1}\rangle=$ & $c_{1}^{\dagger} c_{2}^{\dagger} c_{4}^{\dagger}|0\rangle$ & \\
\hline & $c_{1}^{\dagger} c_{4}^{\dagger}|0\rangle$ & \\
& $c_{2}^{\dagger} c_{4}^{\dagger}|0\rangle$ & \\
& $c_{3}^{\dagger} c_{4}^{\dagger}|0\rangle$ & $-s$ \\
& $c_{1}^{\dagger} c_{2}^{\dagger}|0\rangle$ &
\end{tabular}

Importantly, the ground states are two-fold degenerate, and sites 1 and 2 are either both filled, or neither are filled. As a result, the ground states $|\tilde{0}\rangle$ and $|\tilde{1}\rangle$ act as hard-core boson states with boson number $\eta=0$ and $\eta=1$, respectively.

This boson can be thought of as a $p_{z}$-wave Cooper pair, where sites 1 and 2 are displaced in the $z$-direction. If the Cooper pair condenses (in a larger lattice model, e.g. Eq. (5) in the next section), then a nodeless superconducting state will result. By nodeless, we mean that 
the superconducting order parameter does not have any nodal lines in momentum space since there is a finite energy gap [ $\sim s / 2$ from Eq. (3)] to all single-fermion excitations. ${ }^{2}$

If we ignore sites 3 and 4 (e.g. by tracing them out ${ }^{3}$ ), then the ground states and energy gap of sites 1 and 2 can be roughly described by the following two-site effective Hamiltonian with an attractive interaction!

$$
H_{\mathrm{eff}}^{(4)}=-U_{\mathrm{eff}}\left(n_{1}-\frac{1}{2}\right)\left(n_{2}-\frac{1}{2}\right)
$$

where $\frac{1}{2} U_{\text {eff }}=\frac{1}{2} s+O\left(s^{2} / U\right)$ is the energy-gap to the excited states in Eq. (3).

To understand the effective attractive interaction, note that if there is a fermion at site 1 , then the strong repulsion $U$ will prevent the occupation of site 3 . This prevents the hopping term $s$ from contributing to a lower ground state energy. Due to the negative chemical potential, the ground state $\left(c_{1}^{\dagger} c_{2}^{\dagger} c_{4}^{\dagger}|0\rangle\right)$ prefers to fill sites 2 and 4 . Alternatively, if sites 1 and 2 are empty, then a single fermion can resonate between sites 3 and 4, leading to the state $\frac{1}{\sqrt{2}}\left(c_{3}^{\dagger}+c_{4}^{\dagger}\right)|0\rangle$. We have tuned $\mu / s$ such that these two cases result in equal-energy ground states. Thus, at low energy, sites 1 and 2 are either both filled or are both empty, which is in accordance with the effective attraction in Eq. (4).

Thus, $H^{(4)}$ [Eq. (2)] demonstrates a simple mechanism for how a fermion hopping model with strong repulsive interactions can lead to an effective attractive interaction.

\section{Minimal Model}

By using $H^{(4)}$ to generate an effective attractive interaction, we can write down a simple repulsive fermion model with a superconducting ground state in the limit of strong interactions. The model is simply a grid of coupled $H^{(4)}$ models:

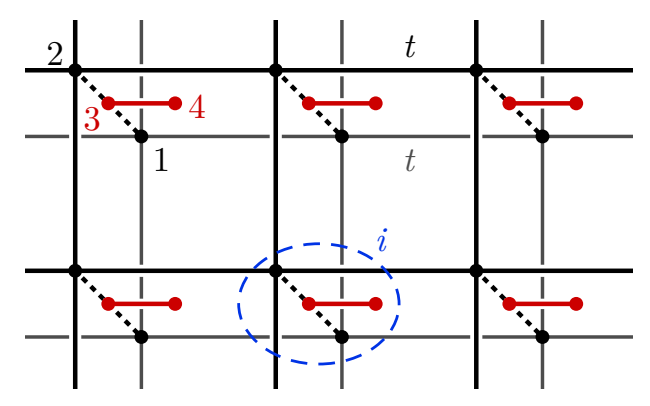

$$
\begin{aligned}
H_{\square}= & H_{\square}^{\prime}+\sum_{i} H_{i}^{(4)} \\
H_{\square}^{\prime}= & -t \sum_{\langle i j\rangle} \sum_{\alpha=1,2}\left(c_{i, \alpha}^{\dagger} c_{j, \alpha}+c_{j, \alpha}^{\dagger} c_{i, \alpha}\right) \\
& -\mu^{\prime} \sum_{i}\left(n_{i, 1}+n_{i, 2}\right) \\
H_{i}^{(4)}= & -s\left(c_{i, 3}^{\dagger} c_{i, 4}+c_{i, 4}^{\dagger} c_{i, 3}\right) \\
& +U\left(n_{i, 1}+n_{i, 2}\right) n_{i, 3}-\mu \sum_{\alpha=1}^{4} n_{i, \alpha}
\end{aligned}
$$

Here, we are considering two coupled two-dimensional square-lattice layers (black). $\sum_{\langle i j\rangle}$ sums over all pairs of nearest-neighbor unit cells $i$ and $j$. Each unit cell $i$ is composed of an $H^{(4)}$ model, which includes four spin-less fermions $c_{i, \alpha}$ indexed by $\alpha=1,2,3,4$. $H_{\square}^{\prime}$ adds a

\footnotetext{
${ }^{2}$ Nodeless superconductivity also appears in the inhomogeneous Hubbard model [33].

${ }^{3}$ If we take the low-temperature $(\beta s \gg 1)$ density matrix $\rho=\frac{1}{Z} e^{-\beta H^{(4)}}$ (where $Z=\operatorname{tr} e^{-\beta H^{(4)}}$ ) of $H^{(4)}$ [Eq. (2)], and trace out sites 3 and 4 , then the resulting density matrix is equal to the density matrix $\rho_{\text {eff }}$ of $H_{\text {eff }}^{(4)}$ (also known as the entanglement Hamiltonian $[41,42]$ ) up to $O\left(e^{-\frac{3}{2} \beta s}\right)$ corrections. That is, $\rho_{\text {eff }}=\operatorname{tr}_{34} \rho=\sum_{n_{1}^{\prime}, n_{2}^{\prime}=0,1}^{n_{1}, n_{2}, n_{3}, n_{4},}\left|n_{1} n_{2}\right\rangle\left\langle n_{1} n_{2} n_{3} n_{4}|\rho| n_{1}^{\prime} n_{2}^{\prime} n_{3} n_{4}\right\rangle\left\langle n_{1}^{\prime} n_{2}^{\prime}\right|+O\left(e^{-\frac{3}{2} \beta s}\right)$
} 
hopping term $t$ and an onsite potential $\mu^{\prime}$ for the $\alpha=1,2$ fermions. We will focus on the following limit:

$$
\left|\mu^{\prime}\right|<t \ll \mu=s / 2 \ll U
$$

In this limit, each $H_{i}^{(4)}$ will approximately always be in one of its two ground states [Eq. (3)]. The fermion hopping term in $H_{\square}^{\prime}$ couples the $H_{i}^{(4)}$ models together. But in order for each $H_{i}^{(4)}$ to remain in its ground state, $H_{\square}^{\prime}$ must act twice in order to move two fermions (a Cooper pair) from one site to another. Thus, we can use degenerate perturbation theory $[43,44]$ to obtain a low-energy effective Hamiltonian at order $t^{2} / s$. (See Appendix B for details.) The resulting model can be written in the form of the following hard-core boson model:

$$
\begin{aligned}
H_{\square}^{\mathrm{eff}}= & -t_{\mathrm{eff}} \sum_{\langle i j\rangle}\left(b_{i}^{\dagger} b_{j}+b_{j}^{\dagger} b_{i}\right)-2 \mu^{\prime} \sum_{i} \eta_{i} \\
& +V_{\mathrm{eff}} \sum_{\langle i j\rangle}\left(\eta_{i}-\frac{1}{2}\right)\left(\eta_{j}-\frac{1}{2}\right)
\end{aligned}
$$

where $V_{\text {eff }}=2 t_{\text {eff }}$ and $t_{\text {eff }}=t^{2} / s$. The hard-core constraint implies that the boson number operator $\eta_{i}=b_{i}^{\dagger} b_{i}=0,1$. ( $H_{\square}^{\text {eff }}$ can also be transformed into an XXZ spin model in a magnetic field ${ }^{4}$.)

Physically, the boson is a Cooper pair of the fermions: $b_{i} \sim c_{i, 1} c_{i, 2}$. The boson hopping term $t_{\text {eff }} b_{i}^{\dagger} b_{j}$ results from a virtual process $\left(t c_{i, 2}^{\dagger} c_{j, 2}\right)\left(t c_{i, 1}^{\dagger} c_{j, 1}\right)$ that hops two fermions from site $j$ to $i$. The nearest-neighbor repulsion $V_{\text {eff }}\left(\eta_{i}-\frac{1}{2}\right)\left(\eta_{j}-\frac{1}{2}\right)$ results from a virtual process $\left(t c_{j, \alpha}^{\dagger} c_{i, \alpha}\right)\left(t c_{i, \alpha}^{\dagger} c_{j, \alpha}\right)$ where a fermion hops from site $j$ to $i$ and then back to $j$. In both virtual processes, the intermediate state has a large energy $s \gg t$, which penalizes the virtual processes and results in the energy scaling $t^{2} / s$ for $t_{\text {eff }}$ and $V_{\text {eff. }}$.

The phase diagram of this effective boson model is shown in Fig. 1. The ground state is in a superfluid phase when $0 \neq\left|\mu^{\prime}\right|<4 t_{\text {eff }}$ (and $V_{\text {eff }}=2 t_{\text {eff }}$ ). Since the effective boson carries the same charge as two fermions, the superfluid in the effective model corresponds to a superconductor in the original fermion model $H_{\square}$ [Eq. (5)]. Therefore, the ground state of $H_{\square}$ is a superconductor in the limit of interest [Eq. (6)] when $0 \neq\left|\mu^{\prime}\right|<4 t^{2} / s$.

\section{Extensions}

Finding a material that realizes the model [Eq. (5)] in the previous section may be difficult. In this section, we will exemplify possible ways that the model can be extended in order to make it more realistic. In Appendix A, we will also explore an alternative lattice geometry. An actual material may realize more than one of these extensions.

\footnotetext{
${ }^{4} H_{\square}^{\text {eff }}$ in Eq. (7) can be viewed as an XXZ spin-1/2 model $H_{\square}^{\text {eff }}=-\frac{1}{2} t_{\text {eff }} \sum_{\langle i j\rangle}\left(\sigma_{i}^{x} \sigma_{i}^{x}+\sigma_{i}^{y} \sigma_{i}^{y}\right)+$ $\frac{1}{4} V_{\text {eff }} \sum_{\langle i j\rangle} \sigma_{i}^{z} \sigma_{i}^{z}-\mu^{\prime} \sum_{i} \sigma_{i}^{z}$ by replacing the hard-core boson operator $b_{j} \rightarrow \frac{1}{2}\left(\sigma_{j}^{x}+i \sigma_{j}^{y}\right)$ with Pauli operators $\sigma_{i}^{\mu}$. Since $V_{\text {eff }}=2 t_{\text {eff }}, H_{\square}^{\text {eff }}$ can also be transformed into an $S U(2)$ anti-ferromagnetic Heisenberg model in an applied field $H_{\square}^{\text {eff }}=\frac{1}{2} t_{\text {eff }} \sum_{\langle i j\rangle} \vec{\sigma}_{i} \cdot \vec{\sigma}_{j}-\mu^{\prime} \sum_{i} \sigma_{i}^{z}$ by rotating the spins on the A sublattice by the unitary operator $U=\prod_{i \in \mathrm{A}} \sigma_{i}^{z}$.
} 


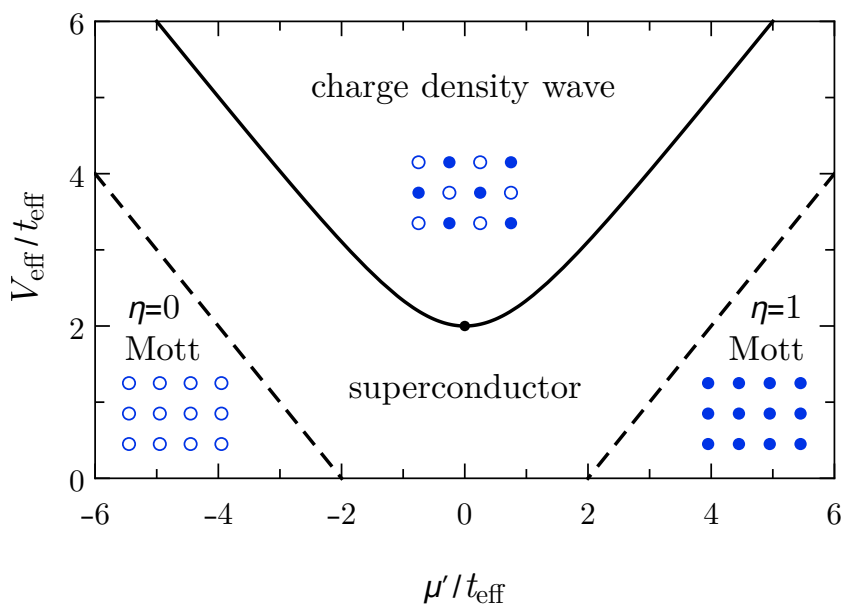

Figure 1: A phase diagram of the hard-core boson model [Eq. (7)] extracted from Ref. [45]. A large boson chemical potential $\mu^{\prime}$ results in a Mott insulating phase with boson number $\eta=0$ or $\eta=1$ on every site. A large boson repulsion $V_{\text {eff }}$ induces a charge density wave where half of the hard-core boson states are filled in a checkerboard pattern. In between these phases is a superconducting phase. The fermion model [Eq. (5)] results in $V_{\text {eff }} / t_{\text {eff }}=2$. The phase transitions across the dashed lines are continuous, while the transition across the solid line is discontinuous [46]. (There is a hidden $S U(2)$ symmetry when $\mu^{\prime}=0$ and $V_{\text {eff }} / t_{\text {eff }}=2$.)

\subsection{Missing Hopping}

The absence of a fermion hopping between sites 3 and 1 (and between 3 and 2) may seem peculiar. Let us then consider the effect of extending the 4-site model [Eq. (2)] with such a hopping:

$$
H_{t^{\prime}}=-t^{\prime}\left(c_{1}^{\dagger} c_{3}+c_{3}^{\dagger} c_{1}\right)-t^{\prime}\left(c_{2}^{\dagger} c_{3}+c_{3}^{\dagger} c_{2}\right)
$$

If the hopping energy $t^{\prime}$ is much less than the energy gap $s / 2$, then the low-energy eigenstates and energies in Eq. (3) will not change. But if $t^{\prime} \gtrsim s / 2$, then the low-energy states and energy spectrum will change significantly, which will likely destroy the superconducting ground state when the 4-site clusters are coupled together.

However, we should also consider the effect of a repulsive interaction between sites 3 and 4, which we will write as:

$$
H_{U^{\prime}}=U^{\prime}\left(n_{3}+n_{4}-1\right)^{2}
$$

Since $U$ is large, it is natural to also consider the possibility that $U^{\prime}$ is also large. In this case, $U^{\prime}$ energetically forbids states that do not have a total of one fermion on sites 3 and 4 . Therefore, if we consider extending the 4 -site model by these terms, $H^{(4)}+H_{t^{\prime}}+H_{U^{\prime}}$ [Eqs. (2), (8), (9)], then this extended model will have the same low-energy eigenstates and energies in Eq. (3) as the original 4-site model as long as $t^{\prime} \ll \max \left(s, U^{\prime}\right)$ (and $\mu=s / 2$ and $s \ll U$ as before). Therefore, when the 4 -site clusters are coupled together, the additional hopping $t^{\prime}$ will not hamper superconductivity as long as $t^{\prime} \ll \max \left(s, U^{\prime}\right)$. 


\subsection{Covalent Bonds}

The geometry of the 4-site cluster [Eq. (2)] may seem unnatural in a material realization due to site 4 , which only couples to a single site. However, the cluster can be expanded such that the middle section models a covalent bond between two or more ions (or nuclei).

We will now discuss the example involving a covalent bond between two ions, which can be modelled by the following 5 -site cluster:

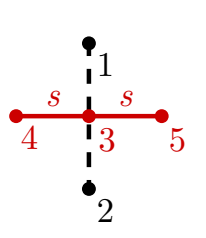

$$
\begin{aligned}
H^{(5)}= & -s\left(c_{3}^{\dagger} c_{4}+c_{4}^{\dagger} c_{3}\right) \\
& -s\left(c_{3}^{\dagger} c_{5}+c_{5}^{\dagger} c_{3}\right) \\
& +U\left(n_{1}+n_{2}\right) n_{3} \\
& -\mu\left(n_{1}+n_{2}+n_{3}+n_{4}\right)
\end{aligned}
$$

$c_{\alpha}$ are five spinless fermion annihilation operators with site/orbital index $\alpha=1,2,3,4,5$. Sites 3 , 4, and 5 model a covalent bond between a pair of ions at sites 4 and 5 . Site 3 is (very coarsely) modelling the electron states between the two ions. One could think of sites 4 and 5 as $s$ orbitals, while site 3 can be thought of as the superposition of $p_{x}$ orbitals of ions 3 and 4 that constructively interferes in the area between the ions. However, other combinations of orbitals are also possible.

When $\mu=s / \sqrt{2}$ and $s \ll U$, the two lowest energy levels are:

$$
\begin{array}{c|c}
|\psi\rangle & E \\
\hline|\tilde{0}\rangle=\frac{1}{2 \sqrt{2}}\left(c_{i, 4}^{\dagger}+\sqrt{2} c_{i, 3}^{\dagger}+c_{i, 5}^{\dagger}\right)\left(c_{i, 4}^{\dagger}-c_{i, 5}^{\dagger}\right)|0\rangle & -2 \sqrt{2} s \\
|\tilde{1}\rangle=c_{i, 1}^{\dagger} c_{i, 2}^{\dagger} c_{i, 4}^{\dagger} c_{i, 5}^{\dagger}|0\rangle & \\
\hline 6 \text { degenerate states } & -\frac{3}{2} \sqrt{2} s
\end{array}
$$

Similar to the 4-site model [Eq. (3)], the ground states are two-fold degenerate and act as hard-core boson states. When an electron is at site 1 or 2 , the covalent bond is damaged since the repulsive interaction $U$ prevents fermions from hopping onto site 3 . In the covalent bond picture, the covalent bond mediates an effective attractive interaction [of the form of Eq. (4)] between the fermions on sites 1 and 2, and a filled hard-boson corresponds to a damaged covalent bond.

If many 5-site clusters are weakly coupled together in a grid [similar to Eq. (5)], then the low-energy physics can be effectively described by a hard-core boson model [Eq. (7) with $V_{\text {eff }}=2 t_{\text {eff }}$ and $\left.t_{\text {eff }}=t^{2} / \sqrt{2} s\right]$. A superconducting ground state results when $0 \neq\left|\mu^{\prime}\right|<4 t_{\mathrm{eff}}=2 \sqrt{2} t^{2} / s$, where $\mu^{\prime}$ is the onsite potential for the fermions at sites 1 and 2 [just as in Eq. (5)].

\subsection{Spinful Fermions}

The previous models have all involved spinless fermions. But electrons are spin-half particles. In this section, we will show that if a spin degree of freedom is added to the fermions in the 4-site model, then a superconducting ground state may still result as long as a large on-site Hubbard repulsion is included. Alternatively, an applied magnetic field could gap out the spin degree of freedom and effectively result in the spinless fermion model in Sec. 3. 
A spinful generalization of the 4-site model is:

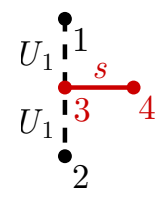

$$
\begin{aligned}
H^{\text {spin }}= & -s \sum_{\sigma=\uparrow, \downarrow}\left(c_{3, \sigma}^{\dagger} c_{4, \sigma}+c_{4, \sigma}^{\dagger} c_{3, \sigma}\right) \\
& +U_{0} \sum_{\alpha=1,2} n_{\alpha}\left(n_{\alpha}-1\right) \\
& +U_{1}\left(n_{1}+n_{2}\right) n_{3} \\
& -\mu_{12}\left(n_{1}+n_{2}\right)-\mu_{34}\left(n_{3}+n_{4}\right)
\end{aligned}
$$

where $n_{\alpha}=\sum_{\sigma} n_{\alpha, \sigma}$ is the total fermion number on site $\alpha$ and $\sigma=\uparrow, \downarrow$ denotes to two electron spin states.

When $\mu_{12}=2 \mu_{34}=s, s \ll U_{0}$, and $s \ll U_{1}$, the lowest energy levels are:

$$
\begin{array}{c|c}
|\psi\rangle & E \\
\hline|\tilde{0}\rangle=\frac{1}{2}\left(c_{3 \uparrow}^{\dagger}+c_{4 \uparrow}^{\dagger}\right)\left(c_{3 \downarrow}^{\dagger}+c_{4 \downarrow}^{\dagger}\right)|0\rangle & -3 s \\
\left|\tilde{1}_{\sigma \sigma^{\prime}}\right\rangle=c_{1, \sigma}^{\dagger} c_{2, \sigma^{\prime}}^{\dagger} c_{4 \uparrow}^{\dagger} c_{4 \downarrow}^{\dagger}|0\rangle & \\
\hline 10 \text { degenerate states } & -\frac{5}{2} s
\end{array}
$$

The ground states are five-fold degenerate. $\left|\tilde{1}_{\sigma \sigma^{\prime}}\right\rangle$ denotes four different spin states indexed by $\sigma, \sigma^{\prime}=\uparrow, \downarrow$. Therefore, the low-energy states behave like a hard-core boson with four different spin states.

If many spinful 4-site clusters are coupled together in a grid [similar to Eq. (5)], then the low-energy physics can be effectively described by a hard-core boson model [similar to Eq. (7)] where the boson has four spin states. It is feasible that this hard-core boson model has a superconducting ground state in some regions of its phase diagram. However, additional perturbations should be added to the model since they will generically split the degeneracy between the spin singlet and triplet states of the hard-core boson.

\section{Discussion}

We have considered a simple two-dimensional lattice model [Eq. (5)] with a superconducting ground state. At temperatures below the single-particle fermion gap $s / 2$ [from Eq. (3)], the model is well-approximated by a hard-core boson model [Eq. (7)] with hopping strength $t_{\text {eff }} \sim t^{2} / s$. If we consider a 3D stack of the $2 \mathrm{D}$ model with a weak fermion hopping between the stacks, then the resulting three-dimensional model can be expected ${ }^{5}$ to exhibit superconductivity at temperatures below $T_{\mathrm{c}} \sim t_{\mathrm{eff}} / 2 \sim t^{2} / 2 s$ [45]. Although we only considered a single corner [Eq. (6)] of the phase diagram, any sufficiently-small local perturbation can be added without destroying the superconductivity.

The Cooper pairing is ultimately a result of the local Coulomb repulsion physics in the 4-site fermion model [Eq. (2)], and the size of the Cooper pair is just a single unit cell. Because the Cooper pairing results from charge interactions, it is interesting to note that the superconducting phase neighbors a charge density wave order (Fig. 1).

\footnotetext{
${ }^{5}$ Before coupling the stacks, each layer is in a state with quasi-long-range superconducting order below a Berezinskii-Kosterlitz-Thouless transition [47] critical temperature $T_{\mathrm{KT}} \sim t_{\mathrm{eff}}$. Since the correlation length of each layer is infinite, a weak fermion coupling between the layers will result in a long-range superconducting order with roughly the same critical temperature $T_{\mathrm{c}} \sim T_{\mathrm{KT}}$.
} 
At temperatures above the superconducting critical temperature $T_{\mathrm{c}}$ but below $T^{\star} \sim s$, the fermions are Cooper paired with a gap $\Delta \approx s / 2$ to single-fermion excitations. Ref. [48] showed that the DC (zero frequency) resistivity of the hard-core boson model [Eqs. (7)] with $\mu^{\prime}=V_{\text {eff }}=0$ is ${ }^{6}$

$$
\rho \approx 0.23 \frac{h}{4 e^{2}} k_{\mathrm{B}} T / t_{\mathrm{eff}}
$$

at high temperatures, which could apply to our fermion model $H_{\square}$ [Eq. (5)] in the temperature range $t_{\text {eff }} \ll T \ll s$. This regime of a large single-particle gap and large resistivity (linear in temperature) therefore appears to be an analog of the pseudo-gap physics [49-52] seen in the cuprate and iron-based superconductors. However, Ref. [48] only considered a hard-core boson model at half filling and without a nearest-neighbor Hubbard repulsion $\left(\mu^{\prime}=V_{\text {eff }}=0\right)$. Future work is required to determine the robustness of the large linear resistivity $(\rho \propto T)$ to these perturbations, which are present in our low-energy boson models.

It could also be interesting to investigate the physics above temperature $T^{\star} \sim s$. One possibility is a crossover to strange/bad metal physics [53-62], i.e. a large resistivity linear in temperature without a large single-particle gap.

As discussed in Sec. 4, the model can be extended in various ways that could help facilitate a material realization. For example, it is possible to view the fermions on sites 3 and 4 as an effective covalent bond between ions (which could be modeled using more than just two sites). In this picture, the presence of a fermion at site 1 or 2 damages the covalent bond by imposing a large Coulombic energy repulsion, which ultimately leads to Cooper pairing. Furthermore, the effective spinless fermion model [Eq. (5)] could result from an applied (or induced) in-plane magnetic field, which can gap out the spin degree of freedom. Such an example is of practical interest since it can result in a superconductor that is more robust to strong magnetic fields.

Another possibility is to think of the 4-site model [Eq.(2)] as a minimal model for a molecule [63]. If a molecule with similar physics can be discovered, then a liquid or crystal of such molecules could exhibit superconductivity. In particular, the lowest-energy states of the molecule should have fermion occupation numbers that differ by even integers (with at least one of them non-zero), as in Eq. (3). In fact, this kind of physics has already been shown to occur in doped buckminsterfullerene $C_{60}$ molecules [64-66]. Alternatively, one can view the 4-site model as a simplified toy model for a recent carbon nanotube experiment [39].

Hard-core boson models have been shown to emerge in certain repulsive fermions models in previous work $[35,36]$. These works considered inhomogeneous spin-half Hubbard models, which were motivated by existing materials. Our motivation differed in that our objective was to find the simplest possible theoretical model of unconventional superconductivity without a bias towards existing materials. The advantage of this approach is that it helps to elucidate the simplest possible mechanism for Cooper pairing.

We hope that our model will help improve our understanding of unconventional superconductivity and inspire new candidate superconducting materials. In particular, we hope that it will be possible to construct layered materials that are engineered to mimic models similar to the models discussed in this work. This may be feasible since the superconducting critical temperature $T_{c}$ of our models is relatively easy to predict, which could lead to material guidelines for a higher $T_{c}$ in certain cases.

\footnotetext{
${ }^{6}$ This resistivity is obtained from Eq. (75) of Ref. [48]. $k_{\mathrm{B}}$ is Boltzmann's constant, and $h / 4 e^{2}$ is the quantum of resistance for a charge $q=2 e$ Cooper pair, where $h$ is Planck's constant.
} 


\section{Acknowledgements}

We thank Arun Paramekanti, Alex Thomson, Jong Yeon Lee, Arbel Haim, Leonid Isaev, and Olexei Motrunich for helpful discussions.

Funding information This work was supported by the NSERC of Canada and the Center for Quantum Materials at the University of Toronto. KS also acknowledges support from the Walter Burke Institute for Theoretical Physics at Caltech.

\section{A Bilayer Triangular Lattice}

In this appendix, we will exemplify another nontrivial way in which the minimal model in Sec. 3 can be modified so as to have a lattice structure that is more likely to have a material realization.

We will consider the following 6 -site spinless fermion model:

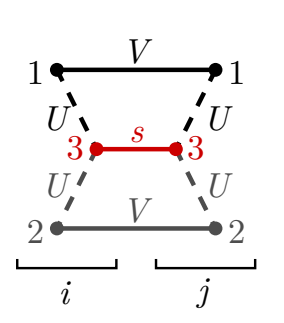

$$
\begin{aligned}
H_{i j}^{(6)}= & -s\left(c_{i, 3}^{\dagger} c_{j, 3}+c_{j, 3}^{\dagger} c_{i, 3}\right) \\
& +U \sum_{i^{\prime}=i, j}\left(n_{i^{\prime}, 1}+n_{i^{\prime}, 2}\right) n_{i^{\prime}, 3} \\
& +V\left(n_{i, 1} n_{j, 1}+n_{i, 2} n_{j, 2}\right) \\
& -\mu \sum_{i^{\prime}=i, j}\left(n_{i^{\prime}, 1}+n_{i^{\prime}, 2}+n_{i^{\prime}, 3}\right)
\end{aligned}
$$

$i$ and $j$ index different 3 -site clusters, while $\alpha=1,2,3$ indexes the three sites within a cluster. $H_{i j}^{(6)}$ couples two 3 -site clusters $(i$ and $j$ ) together.

When $V=\mu=s / 2$ and $s \ll U$, the two lowest energy levels of $H_{i j}^{(6)}$ are:

$$
\begin{aligned}
& \begin{array}{c|c}
|\psi\rangle & E \\
\hline|\widetilde{00}\rangle=\frac{1}{\sqrt{2}}\left(c_{i, 3}^{\dagger}+c_{j, 3}^{\dagger}\right)|0\rangle &
\end{array} \\
& |\widetilde{10}\rangle=c_{i, 1}^{\dagger} c_{i, 2}^{\dagger} c_{j, 3}^{\dagger}|0\rangle \quad-\frac{3}{2} s \\
& \begin{array}{c|c}
|\widetilde{01}\rangle=c_{j, 1}^{\dagger} c_{j, 2}^{\dagger} c_{i, 3}^{\dagger}|0\rangle & \\
\hline 14 \text { degenerate states } & -s
\end{array}
\end{aligned}
$$

The lowest energy level is now triply degenerate. But since the three low-energy states each differ by an even number of fermions, we can still think of them as hard-core boson states $\left|\widetilde{\eta_{i} \eta_{j}}\right\rangle$ with fillings $\eta_{i}, \eta_{j}=0,1$ but where the $|\widetilde{11}\rangle$ state is gapped out due to a large effective bosonic repulsive interaction. If this effective boson condenses, then a superconducting state will result. 
To achieve this, we will embed $H_{i j}^{(6)}$ into a layered triangular lattice:

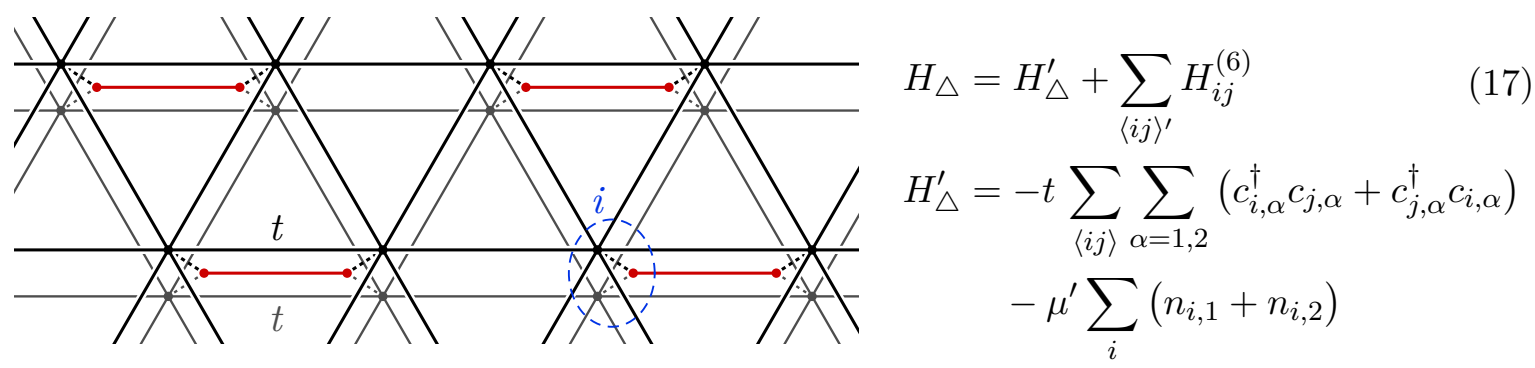

Similar to Eq. (15), $i$ and $j$ index the different 3 -site clusters, which are located at the vertices of a triangular lattice. $\sum_{\langle i j\rangle}$ sums over all nearest-neighbor 3 -site clusters (along the solid gray and black lines), while $\sum_{\langle i j\rangle^{\prime}}$ only sums over the neighboring 3 -site clusters with a red line between them. We will focus on the following limit:

$$
\left|\mu^{\prime}\right|<t \ll V=\mu=s / 2 \ll U
$$

Again, we can use degenerate perturbation theory to derive a low-energy effective hardcore boson model (see Appendix B.2 for details):

$$
\begin{aligned}
H_{\triangle}^{\mathrm{eff}}= & -\frac{t^{2}}{s} \sum_{\langle i j\rangle^{\prime \prime}}\left(b_{i}^{\dagger} b_{j}+b_{j}^{\dagger} b_{i}\right)-2 \mu^{\prime} \sum_{i} \eta_{i} \\
& +\frac{2 t^{2}}{s} \sum_{\langle i j\rangle^{\prime \prime}}\left(1-\eta_{\hat{\imath}}\right) \underbrace{\left(\eta_{i} \eta_{j}-\frac{1}{2} \eta_{i}-\frac{1}{2} \eta_{j}\right)}_{\left(\eta_{i}-\frac{1}{2}\right)\left(\eta_{j}-\frac{1}{2}\right)-\frac{1}{4}}\left(1-\eta_{\hat{\jmath}}\right)
\end{aligned}
$$

constraint: $\eta_{i} \eta_{\hat{\imath}}=0$ across dashed red links

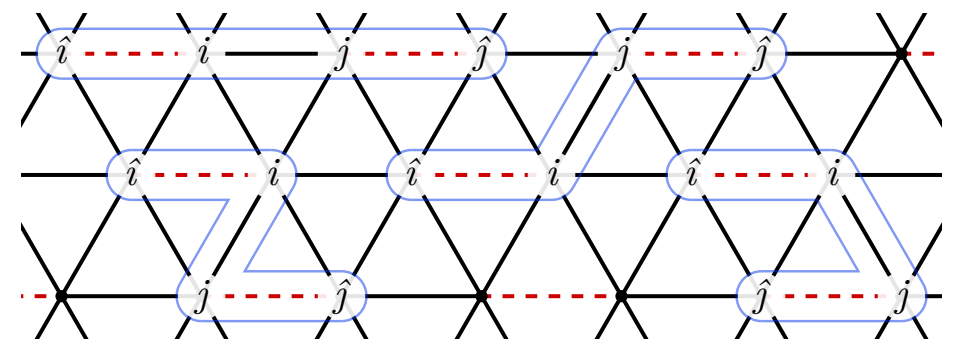

$\sum_{\langle i j\rangle \text { " }}$ sums over neighboring sites across a black link. The last term sums over every pair of neighboring sites $\langle i j\rangle$ across a black link; we then define the $\hat{\imath}$ in $\left(1-\eta_{\hat{\imath}}\right)$ to be the site across the dashed red link from $i$, and similar for $\hat{j}$ and $j$. Above, we highlight four examples in blue of the $(\hat{\imath}, i, j, \hat{\jmath})$ that are summed over. For each $\langle i \hat{\imath}\rangle$ across a dashed red link, a $\eta_{i} \eta_{\hat{\imath}}=0$ constraint results because none of the three low-energy states [Eq. (16)] across a dashed red link correspond to a state with two bosons. The hard-core boson number operator is $\eta_{i}=b_{i}^{\dagger} b_{i}=0,1$. Physically, the boson is a Cooper pair of the fermions: $b_{i} \sim c_{i, 1} c_{i, 2}$.

The last term in $H_{\triangle}^{\text {eff }}$ is a four-boson repulsion term that results from a virtual process $\left(t c_{j, \alpha}^{\dagger} c_{i, \alpha}\right)\left(t c_{i, \alpha}^{\dagger} c_{j, \alpha}\right)$ where a fermion hops across a black link from site $j$ to $i$ and then back to $j$. The projection operators $\left(1-\eta_{\hat{\imath}}\right)\left(1-\eta_{\hat{\jmath}}\right)$ result due to the $\eta_{i} \eta_{\hat{\imath}}=\eta_{j} \eta_{\hat{\jmath}}=0$ constraint, which prevents the virtual process from occurring when $\hat{\imath}$ or $\hat{\jmath}$ is occupied by a boson. At a 
mean-field level, we can think of the projection operators as effectively weakening the repulsive interaction $\left(\eta_{i}-\frac{1}{2}\right)\left(\eta_{j}-\frac{1}{2}\right)$ and shifting the boson chemical potential $\mu^{\prime}$.

Given its similarity to $H_{\square}^{\text {eff }}$ [Eq. (7)] in the previous section, $H_{\triangle}^{\text {eff }}$ is likely to also have a superfluid ground state for certain $\mu^{\prime}$. However, this will have to be checked numerically, which could be done using sign-free ${ }^{7}$ quantum Monte Carlo [67]. This implies that the original model $H_{\triangle}$ [Eq. (17)] is also likely to have a superconducting ground state in the limit considered in Eq. (18) for some range of $\mu^{\prime}$.

\section{B Effective Hamiltonian}

In this appendix, we will use Schrieffer-Wolff degenerate perturbation theory $[43,44]$ to derive the effective Hamiltonians $H_{\square}^{\text {eff }}$ [Eq. (7)] and $H_{\triangle}^{\text {eff }}$ [Eq. (19)].

The input to degenerate perturbation theory is a Hamiltonian, which is the sum of a degenerate Hamiltonian $H_{0}$ and a small perturbation $H_{1}$ to split the degeneracy:

$$
H=H_{0}+H_{1}
$$

A unitary transformation can be perturbatively derived to rotate $H$ into an effective Hamiltonian that acts only on the degenerate ground state space of $H_{0}$ :

$$
H^{\mathrm{eff}}=E_{0}+\mathcal{P} H_{1} \mathcal{P}+\mathcal{P} H_{1} \mathcal{D} H_{1} \mathcal{P}+\cdots
$$

$E_{0}$ is the ground state energy of $H_{0} ; \mathcal{P}$ projects onto the degenerate ground states of $H_{0}$; and

$$
\mathcal{D}=\frac{1-\mathcal{P}}{E_{0}-H_{0}}
$$

projects into the excited states, but with an energy penalty in the denominator. A more thorough review of degenerate perturbation theory can be found in Appendix B of Ref. [68].

\section{B.1 Minimal Model}

To derive $H_{\square}^{\text {eff }}$ in Eq. (7), we define

$$
H_{0}=\sum_{i} H_{i}^{(4)} \quad H_{1}=H_{\square}^{\prime}
$$

where $H_{i}^{(4)}$ and $H_{\square}^{\prime}$ are defined in Eq. (5). We will work using the limit in Eq. (6), and derive $H_{\square}^{\text {eff }}$ up to corrections of order $O\left(t^{4} / s^{3}\right)$ and $O\left(s^{2} / U\right)$.

First, we will define hard-core boson annihilation and number operators that act on the unperturbed ground states [Eq. (3)] as follows:

$$
\begin{aligned}
b_{i}^{\dagger}\left|\tilde{0}_{i}\right\rangle & =\left|\tilde{1}_{i}\right\rangle & \eta_{i}\left|\tilde{0}_{i}\right\rangle & =0 \\
b_{i}\left|\tilde{1}_{i}\right\rangle & =\left|\tilde{0}_{i}\right\rangle & \eta_{i}\left|\tilde{1}_{i}\right\rangle & =\left|\tilde{1}_{i}\right\rangle \\
b_{i}\left|\tilde{0}_{i}\right\rangle=b_{i}^{\dagger}\left|\tilde{1}_{i}\right\rangle & =0 & \eta_{i} & =b_{i}^{\dagger} b_{i}
\end{aligned}
$$

\footnotetext{
${ }^{7} H_{\triangle}^{\text {eff }}$ does not have a sign problem since $-H_{\triangle}^{\text {eff }}$, which appears in the Boltzmann factor $e^{-\beta H_{\triangle}^{\text {eff }}}$, has positive off-diagonal elements when viewed as a matrix in the boson number basis.
} 
The boson operators can be written in terms of the fermions as

$$
\begin{aligned}
& b_{i}=c_{i, 1} c_{i, 2} \frac{1}{\sqrt{2}}\left(c_{i, 3}^{\dagger}+c_{i, 4}^{\dagger}\right)\left(1-n_{i, 3}\right) c_{i, 4} \\
& \eta_{i}=b_{i}^{\dagger} b_{i}=n_{i, 1} n_{n, 2}\left(1-n_{i, 3}\right) n_{i, 4}
\end{aligned}
$$

Within the ground state space of $H_{0}$, into which $\mathcal{P}$ projects, the above can be simplified to

$$
\begin{aligned}
& \mathcal{P} b_{i} \mathcal{P}=\sqrt{2} \mathcal{P} c_{i, 1} c_{i, 2} \mathcal{P} \\
& \mathcal{P} \eta_{i} \mathcal{P}=\mathcal{P} n_{i, 1} n_{n, 2} \mathcal{P}
\end{aligned}
$$

The $\sqrt{2}$ appears in order to cancel the $\frac{1}{\sqrt{2}}$ in $|\tilde{0}\rangle$ [Eq. (3)].

The first non-constant term of $H^{\text {eff }}$ in Eq. (21) is

$$
\begin{aligned}
\mathcal{P} H_{1} \mathcal{P} & =-\mu^{\prime} \sum_{i} \mathcal{P}\left(n_{i, 1}+n_{i, 2}\right) \mathcal{P} \\
& =-2 \mu^{\prime} \sum_{i} \mathcal{P} \eta_{i} \mathcal{P}
\end{aligned}
$$

Eq. (28) results because the grounds states of $H_{0}$ always have $\eta_{i}=n_{i, 1}=n_{i, 2}$,

The next term is given by

$$
\begin{aligned}
\mathcal{P} H_{1} \mathcal{D} H_{1} \mathcal{P}=+t^{2} \sum_{\langle i j\rangle} \sum_{\alpha, \beta=1,2} \mathcal{P}\left(c_{i, \alpha}^{\dagger} c_{j, \alpha}+c_{j, \alpha}^{\dagger} c_{i, \alpha}\right) \mathcal{D}\left(c_{i, \beta}^{\dagger} c_{j, \beta}+c_{j, \beta}^{\dagger} c_{i, \beta}\right) \mathcal{P} \\
=+t^{2} \sum_{\langle i j\rangle} \sum_{\alpha}\left\{\left|\tilde{1}_{i} \tilde{0}_{j}\right\rangle\left\langle\tilde{1}_{i} \tilde{0}_{j}\left|c_{i, \alpha}^{\dagger} c_{j, \alpha} \frac{1-\mathcal{P}}{-s} c_{i, \bar{\alpha}}^{\dagger} c_{j, \bar{\alpha}}\right| \tilde{0}_{i} \tilde{1}_{j}\right\rangle\left\langle\tilde{0}_{i} \tilde{1}_{j}\right|\right. \\
\left.\quad+\left|\tilde{1}_{i} \tilde{0}_{j}\right\rangle\left\langle\tilde{1}_{i} \tilde{0}_{j}\left|c_{i, \alpha}^{\dagger} c_{j, \alpha} \frac{1-\mathcal{P}}{-s} c_{j, \alpha}^{\dagger} c_{i, \alpha}\right| \tilde{1}_{i} \tilde{0}_{j}\right\rangle\left\langle\tilde{1}_{i} \tilde{0}_{j}\right|+(i \leftrightarrow j)\right\} \\
=+t^{2} \sum_{\langle i j\rangle} \sum_{\alpha}\left\{\left|\tilde{1}_{i} \tilde{0}_{j}\right\rangle \frac{1}{-2 s}\left\langle\tilde{0}_{i} \tilde{1}_{j}|+| \tilde{1}_{i} \tilde{0}_{j}\right\rangle \frac{1}{-2 s}\left\langle\tilde{1}_{i} \tilde{0}_{j}\right|+(i \leftrightarrow j)\right\} \\
=-\frac{t^{2}}{s} \sum_{\langle i j\rangle} \mathcal{P}\left[b_{i}^{\dagger} b_{j}+b_{j}^{\dagger} b_{i}-2\left(\eta_{i}-\frac{1}{2}\right)\left(\eta_{j}-\frac{1}{2}\right)+\frac{1}{2}\right] \mathcal{P}
\end{aligned}
$$

In Eq. (30), we are ignoring higher order $O\left(t^{2} / U\right)$ terms. $\left|\tilde{1}_{i} \tilde{0}_{j}\right\rangle\left\langle\tilde{1}_{i} \tilde{0}_{j}\right|$ projects the unit cell $i$ into the state $|\tilde{1}\rangle$ and $j$ into the state $|\tilde{0}\rangle$ [Eq. (3)]. $(i \leftrightarrow j)$ denotes a copy of the expression to its left with $i$ and $j$ interchanged. $\bar{\alpha}=1$ when $\alpha=2$ and $\bar{\alpha}=2$ when $\alpha=1$. Eq. (31) is obtained by calculating an inner product in an 8-fermion Hilbert space. Eq. (32) makes use of the ground state projection operator $\mathcal{P}$ and hard-core boson operators [Eq. (24)]. The sum over $\alpha$ just results in a factor of two.

Adding together Eqs. (28) and (32) reproduces $H_{\square}^{\text {eff }}$ in Eq. (7) up to constant terms, which we ignore in the main text.

\section{B.2 Triangular Model}

To derive $H_{\triangle}^{\text {eff }}$ in Eq. (19), we define

$$
H_{0}=\sum_{\langle i j\rangle^{\prime}} H_{i j}^{(6)} \quad H_{1}=H_{\triangle}^{\prime}
$$


where $H_{i j}^{(6)}$ and $H_{\triangle}^{\prime}$ are defined in Eq. (17). We will work using the limit in Eq. (18), and derive $H_{\triangle}^{\text {eff }}$ up to corrections of order $O\left(t^{4} / s^{3}\right)$ and $O\left(s^{2} / U\right)$.

We will define hard-core boson annihilation and number operators that act on the unperturbed ground states [Eq. (16)] as follows:

$$
\begin{aligned}
& b_{j}^{\dagger}\left|\widetilde{00}_{j}\right\rangle=\left|\widetilde{01}_{j}\right\rangle \quad \eta_{j}\left|\widetilde{00}_{j}\right\rangle=0 \\
& b_{j}\left|\widetilde{01}_{j}\right\rangle=\left|\widetilde{00}_{j}\right\rangle \quad \eta_{j}\left|\widetilde{01}_{j}\right\rangle=\left|\widetilde{01}_{j}\right\rangle \\
& b_{j}^{\dagger}\left|\widetilde{01}_{j}\right\rangle=b_{j}^{\dagger}\left|\widetilde{10}_{j}\right\rangle=0 \quad \eta_{j}\left|\widetilde{10}_{j}\right\rangle=0 \\
& b_{j}\left|\widetilde{00}_{j}\right\rangle=b_{j}\left|\widetilde{10}_{j}\right\rangle=0 \quad \eta_{j}=b_{j}^{\dagger} b_{j}
\end{aligned}
$$

If $\hat{\jmath}$ is the 3 -site cluster across a red link [shown in Eq. (17)] from $j$, then $b_{\hat{\jmath}}$ acts similarly but on the first digit in the ket; e.g. $b_{\hat{\jmath}}^{\dagger}\left|\widetilde{00}_{j}\right\rangle=\left|\widetilde{10}_{j}\right\rangle$. Note that within the above Hilbert space, the following constraint is obeyed: $\eta_{\hat{\jmath}} \eta_{j}=0$. The boson operators can be written in terms of the fermions as

$$
\begin{aligned}
b_{j} & =c_{j, 1} c_{j, 2} \frac{1}{\sqrt{2}}\left(c_{j, 3}^{\dagger}+c_{\hat{\jmath}, 3}^{\dagger}\right)\left(1-n_{j, 3}\right) c_{\hat{\jmath}, 3} \\
\eta_{j} & =b_{j}^{\dagger} b_{j}=n_{j, 1} n_{j, 2}\left(1-n_{j, 3}\right) n_{\hat{\jmath}, 3}
\end{aligned}
$$

Within the ground state space of $H_{0}$, into which $\mathcal{P}$ projects, the above can be simplified to

$$
\begin{aligned}
& \mathcal{P} b_{j} \mathcal{P}=\sqrt{2} \mathcal{P} c_{j, 1} c_{j, 2} \mathcal{P} \\
& \mathcal{P} \eta_{j} \mathcal{P}=\mathcal{P} n_{j, 1} n_{j, 2} \mathcal{P}
\end{aligned}
$$

The first non-constant term of $H^{\text {eff }}$ in Eq. (21) is

$$
\begin{aligned}
\mathcal{P} H_{1} \mathcal{P} & =-\mu^{\prime} \sum_{i} \mathcal{P}\left(n_{i, 1}+n_{i, 2}\right) \mathcal{P} \\
& =-2 \mu^{\prime} \sum_{i} \mathcal{P} \eta_{i} \mathcal{P}
\end{aligned}
$$

Eq. (38) results because the grounds states of $H_{0}$ always have $\eta_{i}=n_{i, 1}=n_{i, 2}$.

The next term is given by

$$
\begin{aligned}
\mathcal{P} H_{1} \mathcal{D} H_{1} \mathcal{P}= & +t^{2} \sum_{\langle i j\rangle} \sum_{\alpha, \beta=1,2} \mathcal{P}\left(c_{i, \alpha}^{\dagger} c_{j, \alpha}+c_{j, \alpha}^{\dagger} c_{i, \alpha}\right) \mathcal{D}\left(c_{i, \beta}^{\dagger} c_{j, \beta}+c_{j, \beta}^{\dagger} c_{i, \beta}\right) \mathcal{P} \\
= & +t^{2} \sum_{\langle i j\rangle^{\prime \prime}} \sum_{\alpha}\left\{\left|\widetilde{01}_{i} \widetilde{00}_{j}\right\rangle\left\langle\widetilde{10}_{i} \widetilde{00}_{j}\left|c_{i, \alpha}^{\dagger} c_{j, \alpha} \frac{1-\mathcal{P}}{-s} c_{i, \bar{\alpha}}^{\dagger} c_{j, \bar{\alpha}}\right| \widetilde{00}_{i} \widetilde{01}_{j}\right\rangle\left\langle\widetilde{00}_{i} \widetilde{01}_{j}\right|\right. \\
& \left.\quad+\left|\widetilde{01}_{i} \widetilde{00}_{j}\right\rangle\left\langle\widetilde{01}_{i} \widetilde{00}_{j}\left|c_{i, \alpha}^{\dagger} c_{j, \alpha} \frac{1-\mathcal{P}}{-s} c_{j, \alpha}^{\dagger} c_{i, \alpha}\right| \widetilde{01}_{i} \widetilde{00}_{j}\right\rangle\left\langle\widetilde{01}_{i} \widetilde{00}_{j}\right|+(i \leftrightarrow j)\right\} \\
= & +t^{2} \sum_{\langle i j\rangle^{\prime \prime}} \sum_{\alpha}\left\{\left|\widetilde{01}_{i} \widetilde{00}_{j}\right\rangle \frac{1}{-2 s}\left\langle\widetilde{00}_{i} \widetilde{01}_{j}|+| \widetilde{01}_{i} \widetilde{00}_{j}\right\rangle \frac{1}{-2 s}\left\langle\widetilde{01}_{i} \widetilde{00}_{j}\right|+(i \leftrightarrow j)\right\} \\
= & -\frac{t^{2}}{s} \sum_{\langle i j\rangle^{\prime \prime}} \mathcal{P}[b_{i}^{\dagger} b_{j}+b_{j}^{\dagger} b_{i}-2\left(1-\eta_{\hat{i}}\right) \underbrace{\left(\eta_{i} \eta_{j}-\frac{1}{2} \eta_{i}-\frac{1}{2} \eta_{j}\right)}_{\left(\eta_{i}-\frac{1}{2}\right)\left(\eta_{j}-\frac{1}{2}\right)-\frac{1}{4}}\left(1-\eta_{\hat{j}}\right)] \mathcal{P}
\end{aligned}
$$


We are neglecting small $O\left(U^{-1}\right)$ terms. In Eq. (40), $\sum_{\langle i j\rangle^{\prime}}$ (and $\sum_{\langle i j\rangle^{\prime \prime}}$ ) sum over the neighboring 3 -site clusters with (and without) a red line between them in Eq. (17). $\left|\widetilde{01}_{i} \widetilde{00}_{j}\right\rangle\left\langle\widetilde{01}_{i} \widetilde{00}_{j}\right|$ projects the 6 -site unit cell $(\hat{\imath}, i)$ into the state $|\widetilde{01}\rangle$ and $(\hat{\jmath}, j)$ into the state $|\widetilde{00}\rangle[$ Eq. (16)]. $\hat{\imath}$ denotes the 3 -site cluster across a red link from $i$ in Eq. (17), and similar for $\hat{\jmath}$ and $j$.

Adding together Eqs. (38) and (42) reproduces $H_{\triangle}^{\text {eff }}$ in Eq. (19) up to constant terms, which we ignore in Appendix A.

\section{References}

[1] B. Keimer, S. A. Kivelson, M. R. Norman, S. Uchida and J. Zaanen, From quantum matter to high-temperature superconductivity in copper oxides, Nature 518(7538), 179-186 (2015), doi:10.1038/nature14165.

[2] M. R. Norman and C. Pépin, The electronic nature of high temperature cuprate superconductors, Reports on Progress in Physics 66(10), 1547 (2003), doi:10.1088/00344885/66/10/R01.

[3] J. Orenstein and A. J. Millis, Advances in the Physics of High-Temperature Superconductivity, Science 288(5465), 468 (2000), doi:10.1126/science.288.5465.468.

[4] P. A. Lee, N. Nagaosa and X.-G. Wen, Doping a Mott insulator: Physics of high-temperature superconductivity, Rev. Mod. Phys. 78, 17 (2006), doi:10.1103/RevModPhys.78.17.

[5] S. Sachdev, Emergent gauge fields and the high-temperature superconductors, Phil. Trans. R. Soc. A 374(2075), 20150248 (2016), doi:10.1098/rsta.2015.0248.

[6] D. N. Basov and T. Timusk, Electrodynamics of high-T $T_{c}$ superconductors, Rev. Mod. Phys. 77, 721 (2005), doi:10.1103/RevModPhys.77.721.

[7] A. Damascelli, Z. Hussain and Z.-X. Shen, Angle-resolved photoemission studies of the cuprate superconductors, Rev. Mod. Phys. 75, 473 (2003), doi:10.1103/RevModPhys.75.473.

[8] M. A. Kastner, R. J. Birgeneau, G. Shirane and Y. Endoh, Magnetic, transport, and optical properties of monolayer copper oxides, Rev. Mod. Phys. 70, 897 (1998), doi:10.1103/RevModPhys.70.897.

[9] F. C. Zhang and T. M. Rice, Effective Hamiltonian for the superconducting Cu oxides, Phys. Rev. B 37, 3759 (1988), doi:10.1103/PhysRevB.37.3759.

[10] C. Proust and L. Taillefer, The remarkable underlying ground states of cuprate superconductors, arXiv:1807.05074 (2018).

[11] J. G. Bednorz and K. A. Müller, Possible highT $T_{c}$ superconductivity in the BaLa-Cu-O system, Zeitschrift für Physik B Condensed Matter 64(2), 189 (1986), doi:10.1007/BF01303701. 
[12] M. K. Wu, J. R. Ashburn, C. J. Torng, P. H. Hor, R. L. Meng, L. Gao, Z. J. Huang, Y. Q. Wang and C. W. Chu, Superconductivity at $93 \mathrm{~K}$ in a new mixed-phase $Y$ Ba-Cu-O compound system at ambient pressure, Phys. Rev. Lett. 58, 908 (1987), doi:10.1103/PhysRevLett.58.908.

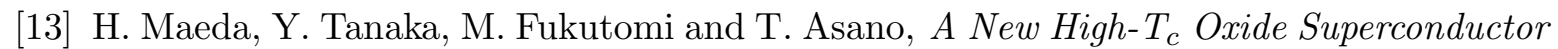
without a Rare Earth Element, Japanese Journal of Applied Physics 27(2A), L209 (1988), doi:10.1143/JJAP.27.L209.

[14] Z. Z. Sheng and A. M. Hermann, Bulk superconductivity at $120 \mathrm{~K}$ in the $T l \Leftrightarrow B a \Leftrightarrow C u \Leftrightarrow O$ system, Nature 332(6160), 138-139 (1988), doi:10.1038/332138a0.

[15] A. Schilling, M. Cantoni, J. D. Guo and H. R. Ott, Superconductivity above $130 \mathrm{~K}$ in the $\mathrm{Hg}-\mathrm{Ba}-\mathrm{Ca}-\mathrm{Cu}-\mathrm{O}$ system, Nature 363(6424), 56-58 (1993), doi:10.1038/363056a0.

[16] S. Raghu, X.-L. Qi, C.-X. Liu, D. J. Scalapino and S.-C. Zhang, Minimal two-band model of the superconducting iron oxypnictides, Phys. Rev. B 77, 220503 (2008), doi:10.1103/PhysRevB.77.220503.

[17] G. R. Stewart, Superconductivity in iron compounds, Rev. Mod. Phys. 83, 1589 (2011), doi:10.1103/RevModPhys.83.1589.

[18] Y. Kamihara, T. Watanabe, M. Hirano and H. Hosono, Iron-Based Layered Superconductor La $\left[O_{1-x} F_{x}\right] F e A s(x=0.05-0.12)$ with $T_{c}=26 \mathrm{~K}$, Journal of the American Chemical Society 130(11), 3296 (2008), doi:10.1021/ja800073m.

[19] Z. A. Ren, J. Yang, W. Lu, W. Yi, G. C. Che, X. L. Dong, L. L. Sun and Z. X. Zhao, Superconductivity at $52 \mathrm{~K}$ in iron based $F$ doped layered quaternary compound $\operatorname{Pr}\left[\mathrm{O}_{1-x} F_{x}\right] \mathrm{FeAs}, \quad$ Materials Research Innovations 12(3), 105 (2008), doi:10.1179/143307508X333686.

[20] G. F. Chen, Z. Li, G. Li, J. Zhou, D. Wu, J. Dong, W. Z. Hu, P. Zheng, Z. J. Chen, H. Q. Yuan, J. Singleton, J. L. Luo et al., Superconducting Properties of the FeBased Layered Superconductor LaFeAsO $O_{0.9} F_{0.1-\delta}$, Phys. Rev. Lett. 101, 057007 (2008), doi:10.1103/PhysRevLett.101.057007.

[21] X. H. Chen, T. Wu, G. Wu, R. H. Liu, H. Chen and D. F. Fang, Superconductivity at 43 $K$ in $\mathrm{SmFeAsO} \mathrm{O}_{1-x} F_{x}$, Nature 453(7196), 761-762 (2008), doi:10.1038/nature07045.

[22] X. Zhu, F. Han, P. Cheng, G. Mu, B. Shen, L. Fang and H.-H. Wen, Superconductivity in fluoride-arsenide $S r_{1-x} L a_{x} F e A s F$ compounds, EPL (Europhysics Letters) 85(1), 17011 (2009), doi:10.1209/0295-5075/85/17011.

[23] T. Hansson, V. Oganesyan and S. Sondhi, Superconductors are topologically ordered, Annals of Physics 313(2), 497 (2004), doi:https://doi.org/10.1016/j.aop.2004.05.006.

[24] W. Kohn and J. M. Luttinger, New Mechanism for Superconductivity, Phys. Rev. Lett. 15, 524 (1965), doi:10.1103/PhysRevLett.15.524.

[25] S. Raghu, S. A. Kivelson and D. J. Scalapino, Superconductivity in the repulsive Hubbard model: An asymptotically exact weak-coupling solution, Phys. Rev. B 81, 224505 (2010), doi:10.1103/PhysRevB.81.224505. 
[26] S. Maiti and A. V. Chubukov, Superconductivity from repulsive interaction, AIP Conference Proceedings 1550(1), 3 (2013), doi:10.1063/1.4818400.

[27] R. Nandkishore, L. S. Levitov and A. V. Chubukov, Chiral superconductivity from repulsive interactions in doped graphene, Nature Physics 8(2), 158-163 (2012), doi:10.1038/nphys2208.

[28] A. Chubukov, Pairing Mechanism in Fe-Based Superconductors, Annual Review of Condensed Matter Physics 3(1), 57 (2012), doi:10.1146/annurev-conmatphys-020911125055 .

[29] M. T. Béal-Monod, C. Bourbonnais and V. J. Emery, Possible superconductivity in nearly antiferromagnetic itinerant fermion systems, Phys. Rev. B 34, 7716 (1986), doi:10.1103/PhysRevB.34.7716.

[30] K. Miyake, S. Schmitt-Rink and C. M. Varma, Spin-fluctuation-mediated evenparity pairing in heavy-fermion superconductors, Phys. Rev. B 34, 6554 (1986), doi:10.1103/PhysRevB.34.6554.

[31] D. J. Scalapino, E. Loh and J. E. Hirsch, d-wave pairing near a spin-density-wave instability, Phys. Rev. B 34, 8190 (1986), doi:10.1103/PhysRevB.34.8190.

[32] P. Monthoux, A. V. Balatsky and D. Pines, Toward a theory of high-temperature superconductivity in the antiferromagnetically correlated cuprate oxides, Phys. Rev. Lett. 67, 3448 (1991), doi:10.1103/PhysRevLett.67.3448.

[33] W.-F. Tsai, H. Yao, A. Läuchli and S. A. Kivelson, Optimal inhomogeneity for superconductivity: Finite-size studies, Phys. Rev. B 77, 214502 (2008), doi:10.1103/PhysRevB.77.214502.

[34] E. Arrigoni, E. Fradkin and S. A. Kivelson, Mechanism of high-temperature superconductivity in a striped Hubbard model, Phys. Rev. B 69, 214519 (2004), doi:10.1103/PhysRevB.69.214519.

[35] H. Yao, W.-F. Tsai and S. A. Kivelson, Myriad phases of the checkerboard Hubbard model, Phys. Rev. B 76, 161104 (2007), doi:10.1103/PhysRevB.76.161104.

[36] L. Isaev, G. Ortiz and C. D. Batista, Superconductivity in Strongly Repulsive Fermions: The Role of Kinetic-Energy Frustration, Phys. Rev. Lett. 105, 187002 (2010), doi:10.1103/PhysRevLett.105.187002.

[37] J. Eroles, G. Ortiz, A. V. Balatsky and A. R. Bishop, Inhomogeneity-induced superconductivity?, EPL (Europhysics Letters) 50(4), 540 (2000), doi:10.1209/epl/i200000303-0.

[38] W. A. Little, Possibility of Synthesizing an Organic Superconductor, Phys. Rev. 134, A1416 (1964), doi:10.1103/PhysRev.134.A1416.

[39] A. Hamo, A. Benyamini, I. Shapir, I. Khivrich, J. Waissman, K. Kaasbjerg, Y. Oreg, F. v. Oppen and S. Ilani, Electron attraction mediated by Coulomb repulsion, Nature 535(7612), 395-400 (2016), doi:10.1038/nature18639. 
[40] D. Allender, J. Bray and J. Bardeen, Model for an Exciton Mechanism of Superconductivity, Phys. Rev. B 7, 1020 (1973), doi:10.1103/PhysRevB.7.1020.

[41] I. Peschel and V. Eisler, Reduced density matrices and entanglement entropy in free lattice models, Journal of Physics A: Mathematical and Theoretical 42(50), 504003 (2009), doi:10.1088/1751-8113/42/50/504003.

[42] X. Wen, S. Ryu and A. W. W. Ludwig, Evolution operators in conformal field theories and conformal mappings: Entanglement Hamiltonian, the sine-square deformation, and others, Phys. Rev. B 93, 235119 (2016), doi:10.1103/PhysRevB.93.235119.

[43] S. Bravyi, D. P. DiVincenzo and D. Loss, Schrieffer-Wolff transformation for quantum many-body systems, Annals of Physics 326(10), 2793 (2011), doi:10.1016/j.aop.2011.06.004.

[44] J. R. Schrieffer and P. A. Wolff, Relation between the Anderson and Kondo Hamiltonians, Phys. Rev. 149, 491 (1966), doi:10.1103/PhysRev.149.491.

[45] G. Schmid, S. Todo, M. Troyer and A. Dorneich, Finite-Temperature Phase Diagram of Hard-Core Bosons in Two Dimensions, Phys. Rev. Lett. 88, 167208 (2002), doi:10.1103/PhysRevLett.88.167208.

[46] F. Hébert, G. G. Batrouni, R. T. Scalettar, G. Schmid, M. Troyer and A. Dorneich, Quantum phase transitions in the two-dimensional hardcore boson model, Phys. Rev. B 65, 014513 (2001), doi:10.1103/PhysRevB.65.014513.

[47] J. M. Kosterlitz and D. J. Thouless, Ordering, metastability and phase transitions in two-dimensional systems, Journal of Physics C: Solid State Physics 6(7), 1181 (1973), doi:10.1088/0022-3719/6/7/010.

[48] N. H. Lindner and A. Auerbach, Conductivity of hard core bosons: A paradigm of a bad metal, Phys. Rev. B 81, 054512 (2010), doi:10.1103/PhysRevB.81.054512.

[49] T. Timusk and B. Statt, The pseudogap in high-temperature superconductors: an experimental survey, Reports on Progress in Physics 62(1), 61 (1999), doi:10.1088/0034$4885 / 62 / 1 / 002$.

[50] Y. Ando, S. Komiya, K. Segawa, S. Ono and Y. Kurita, Electronic Phase Diagram of High-T $T_{c}$ Cuprate Superconductors from a Mapping of the In-Plane Resistivity Curvature, Phys. Rev. Lett. 93, 267001 (2004), doi:10.1103/PhysRevLett.93.267001.

[51] C. Renner, B. Revaz, J.-Y. Genoud, K. Kadowaki and O. Fischer, Pseudogap Precursor of the Superconducting Gap in Under- and Overdoped $\mathrm{Bi}_{2} \mathrm{Sr}_{2} \mathrm{CaCu}_{2} \mathrm{O}_{8+\delta}$, Phys. Rev. Lett. 80, 149 (1998), doi:10.1103/PhysRevLett.80.149.

[52] E. Fradkin, S. A. Kivelson and J. M. Tranquada, Colloquium: Theory of intertwined orders in high temperature superconductors, Rev. Mod. Phys. 87, 457 (2015), doi:10.1103/RevModPhys.87.457.

[53] O. Gunnarsson, M. Calandra and J. E. Han, Colloquium: Saturation of electrical resistivity, Rev. Mod. Phys. 75, 1085 (2003), doi:10.1103/RevModPhys.75.1085. 
[54] E. Perepelitsky, A. Galatas, J. Mravlje, R. Žitko, E. Khatami, B. S. Shastry and A. Georges, Transport and optical conductivity in the Hubbard model: A high-temperature expansion perspective, Phys. Rev. B 94, 235115 (2016), doi:10.1103/PhysRevB.94.235115.

[55] C. H. Mousatov, I. Esterlis and S. A. Hartnoll, Bad metallic transport in a modified Hubbard model, arXiv:1803.08054 (2018).

[56] Y. Werman and E. Berg, Mott-Ioffe-Regel limit and resistivity crossover in a tractable electron-phonon model, Phys. Rev. B 93, 075109 (2016), doi:10.1103/PhysRevB.93.075109.

[57] X.-Y. Song, C.-M. Jian and L. Balents, Strongly Correlated Metal Built from Sachdev-Ye-Kitaev Models, Phys. Rev. Lett. 119, 216601 (2017), doi:10.1103/PhysRevLett.119.216601.

[58] D. Chowdhury, Y. Werman, E. Berg and T. Senthil, Translationally invariant non-Fermi liquid metals with critical Fermi-surfaces: Solvable models, arXiv:1801.06178 (2018).

[59] S. Lederer, Y. Schattner, E. Berg and S. A. Kivelson, Superconductivity and non-Fermi liquid behavior near a nematic quantum critical point, Proceedings of the National Academy of Sciences 114(19), 4905 (2017), doi:10.1073/pnas.1620651114.

[60] S. Martin, A. T. Fiory, R. M. Fleming, L. F. Schneemeyer and J. V. Waszczak, Normalstate transport properties of $\mathrm{Bi}_{2+x} \mathrm{Sr}_{2-y} \mathrm{CuO}_{6+\delta}$ crystals, Phys. Rev. B 41, 846 (1990), doi:10.1103/PhysRevB.41.846.

[61] Y. Mizuguchi, F. Tomioka, S. Tsuda, T. Yamaguchi and Y. Takano, Substitution Effects on FeSe Superconductor, Journal of the Physical Society of Japan 78(7), 074712 (2009), doi:10.1143/JPSJ.78.074712.

[62] B. Lee, S. Khim, J. S. Kim, G. R. Stewart and K. H. Kim, Single-crystal growth and superconducting properties of LiFeAs, EPL (Europhysics Letters) 91(6), 67002 (2010), doi:10.1209/0295-5075/91/67002.

[63] J. E. Hirsch and D. J. Scalapino, Double-valence-fluctuating molecules and superconductivity, Phys. Rev. B 32, 5639 (1985), doi:10.1103/PhysRevB.32.5639.

[64] S. Chakravarty, M. P. Gelfand and S. Kivelson, Electronic Correlation Effects and Superconductivity in Doped Fullerenes, Science 254(5034), 970 (1991), doi:10.1126/science.254.5034.970.

[65] A. F. Hebard, M. J. Rosseinsky, R. C. Haddon, D. W. Murphy, S. H. Glarum, T. T. M. Palstra, A. P. Ramirez and A. R. Kortan, Superconductivity at $18 \mathrm{~K}$ in potassium-doped C60, Nature 350(6319), 600-601 (1991), doi:10.1038/350600a0.

[66] M. J. Rosseinsky, A. P. Ramirez, S. H. Glarum, D. W. Murphy, R. C. Haddon, A. F. Hebard, T. T. M. Palstra, A. R. Kortan, S. M. Zahurak and A. V. Makhija, Superconductivity at $28 \mathrm{~K}$ in $\mathrm{Rb}_{x} \mathrm{C}_{60}$, Phys. Rev. Lett. 66, 2830 (1991), doi:10.1103/PhysRevLett.66.2830. 
[67] A. W. Sandvik, Computational Studies of Quantum Spin Systems, AIP Conference Proceedings 1297(1), 135 (2010), doi:10.1063/1.3518900.

[68] K. Slagle and Y. B. Kim, Fracton topological order from nearest-neighbor two-spin interactions and dualities, Phys. Rev. B 96, 165106 (2017), doi:10.1103/PhysRevB.96.165106. 